was significantly reduced by fumigation with $0.1-0.2 \mathrm{ppm} \mathrm{SO}_{2}$ after $24 \mathrm{~h}$ at both 10 and $20 \mathrm{C}$ (Magan \& McLeod 1988). Germination of filamentous fungi was less affected, although germ tube extension was. In field experiments populations of Sporobolomyces roseus Kluyver \& van Niel on leaves of ripening barley were significantly reduced by exposure to $0.04-0.05 \mathrm{ppm} \mathrm{SO}$. By contrast, those of Aureobasidium pullulans (de Bary) Arnaud were significantly higher compared to unfumigated controls.

These results suggest that some species may be used as natural indicators of pollution levels because of their sensitivity to pollutants, e.g. yeasts. The stimulation or inhibition of components of the phylloplane mycoflora on leaves and ears of ripening cereals could result in a change in the ecological balance on plant surfaces and have consequences for the development of fungal pathogens and growth and yield of the crop.

\title{
References
}

Magan, N. \& Lacey, J. 1986. The phylloplane microflora of ripening wheat and effect of late fungicide application. Annals of Applied Biology 109, 117-128.

Magan, N. \& McLeod, A. R. 1988. In vitro growth and germination of phylloplane fungi in atmospheric sulphur dioxide. Transactions of the British Mycological Society 90, 571-575.

McLeod, A. R., Fackrell, J. E. \& Alexander, K. 1985. Open-air fumigation of field crops: criteria and design for a new experimental system. Atmospheric Environment 19, 1639-1649.

\section{The role of VA mycorrhizas in soil disturbance - included reduction of phosphorus absorption by young maize plants}

\author{
D. G. Evans \\ Department of Land Resource Science, The University of Guelph, Guelph, \\ Ontario, Canada
}

This study was designed to test the hypothesis that disruption of the macro-structure of a previously zero-tilled soil is capable of reducing VA-mycorrhizal infection to a degree such that $\mathrm{P}$ absorption is reduced. Undisturbed soil cores (within plastic cylinders) were excavated from long-term, zero-tilled plots. Soil removed from these plots, which was first subjected to structurally disruptive forces and then packed into identical cylinders, formed the disturbed comparisons. Maize (Zea mays L.), wheat (Triticum aestivum L.), spinach (Spinacia oleracea L.) or rape (Brassica napus L.) were grown in the cores, and $\mathrm{P}$ and $\mathrm{Zn}$ absorption patterns monitored.

Soil disturbance significantly reduced $\mathrm{P}$ and $\mathrm{Zn}$ absorption by maize grown in soil originating from three sites differing in local geography and (or) texture. Disturbance also reduced mycorrhizal infection in the three soils. A significant effect of disturbance upon the VA-mycorrhizal infection of both maize and wheat roots (both mycorrhizal), and also upon the $\mathrm{P}$ absorption by these species, was observed. None was found with respect to spinach and rape comparisons (non mycorrhizal). Injection of benomyl, a potent inhibitor of mycorrhizal fungi, into the soil surface significantly reduced the influence of soil disturbance upon $\mathrm{P}$ absorption. No significant differences were found in VA-mycorrhizal infection within fungicide-treated cores. 
The degree of coincidence in the development of VAM-infection intensity and phosphorus-absorption differences was determined in a study whereby the disturbance effect was established from an initially disturbed soil. These measurements were made on plants which had grown in disturbed soil which was then allowed to remain undisturbed over several cycles of corn growth, and also on plants grown in soil which was repeatedly disturbed. A coincidental evolution of mycorrhizal infection intensity and phosphorus-absorption differences between disturbed and undisturbed treatments was found. Such a correlation is a requirement of our hypothesis. We accepted our hypothesis based upon the above evidence.

\title{
A new automatic electrolytic respirometer
}

\author{
H. T. Tribe \\ Department of Applied Biology, The University, Pembroke Street, Cambridge \\ CB2 3DX, U.K. \\ and

\section{P. Maynard} \\ Institute of Biotechnology, The University, Downing Street, Cambridge CB2 3EF, \\ U.K.
}

The electrolytic method for measuring oxygen uptake in biological systems has a long history. Macfadyen (1961) states that it goes back to 1923, when Fernandes used it for studies with germinating peas. The method has been used in many forms, most of which have been difficult to adapt to more than a few replicates and have required a great deal of attention. For these reasons its full potential has not yet been realised. The basic principle is the enclosure of a biological system within an airspace which is connected to the anodic limb of an electrolysis cell suitable for generating oxygen. Uptake of oxygen by the system reduces the pressure in the airspace such that electrical contact is made between the anode and the electrolyte, and oxygen is supplied to the system. When pressure is restored in the airspace, contact between the anode and electrolyte is broken. Further oxygen uptake causes contact to be made again, and the cycle repeats. Carbon dioxide is absorbed in a vessel of alkali placed within the system and has only a transient influence on pressure in the airspace. The temperature of the system must be kept constant.

The volume of oxygen supplied to the system is measured in one of two ways. The simpler to arrange, but cumbersome and impossible to automate, is the collection of hydrogen gas from the cathodic limb of the electrolysis cell. This has the further disadvantage that this limb must be "open" to the atmosphere and barometric pressure must be taken into consideration. The better alternative is to measure the current required to supply the oxygen and to calculate, from Faraday's laws, the oxygen delivered. Combinations of ammeters and recorders have been used, but 